\title{
ON THE ORIENTATION OF MAGNETIC FIELDS IN QUIESCENT PROMINENCES
}

\author{
ULRICH ANZER* and E. TANDBERG-HANSSEN \\ High Altitude Observatory, National Center for Atmospheric Research**, Boulder, Colo., U.S.A.
}

\section{The Observations and Their Reduction}

The longitudinal component of the magnetic field in quiescent prominences has been measured directly with magnetographs using the Zeeman effect on selected spectral lines (Rust, 1966; Ioshpa, 1968; Harvey, 1969). We know that as a general rule the magnetic field enters the, largely-vertical, sheet-like quiescent prominence on one side and exits on the other. The field traverses the prominence plasma with components both along and at right angles to the long axis of the prominence. It is the purpose of this paper to describe observations that may indicate the relative importance of the two components of the magnetic field, and to derive a distribution function for the magnetic field vectors.

The data for the longitudinal component of the magnetic field, $B_{\|}$, are based on observations of about 70 quiescent prominences studied with the Climax magnetograph during the period 1968-69 (see the paper Tandberg-Hanssen: 'Observations of Magnetic Felds in Quiscent Prominences', this volume, p. 192). We included only those prominences for which the approximation of a plane-parallel sheet seemed satisfactory. Let us consider an orthogonal coordinate system with $x$-axis horizontal and perpendicular to the (idealized) plane prominence sheet, $y$-axis horizontal and along the filament and $z$-axis perpendicular to the photosphere. The observations of a prominence on the solar limb near the equator and in the plane of the sky give $B_{\|}=B_{x}$, see Figure 1, prominence 1. In the more general case, as a prominence at latitude $\phi$ and not in the plane of the sky move onto the disk and toward the central meridian, we have measured the angle $\theta_{1}$ that the assumed vertical plane filament makes with the north-south direction on the Sun, see Figure 1, prominence 2. Figure 2 shows how the observed longitudinal magnetic field, $B_{\|}$, for a given prominence depends on this angle between the $y$-axis and the north-south direction. We emphasize the uncertainties involved in determining this angle for any filament, but taken at face value Figure 2 indicates that $B_{\|}$increases with increasing angle $\theta_{1}$; in other words $B_{y}>B_{x}$.

The procedure outlined to determine the angle $\theta_{1}$ is valid only if the filament does not change in shape or orientation during the time from its limb passage until observed near the central meridian. Furthermore, this measurement is only good up to a certain latitude, as the following discussion will show.

Let $\theta_{1}$ be the observed angle at solar latitude $\phi$. Then, the corresponding angle $\theta$,

* On leave from Max-Planck-Institut für Physik und Astrophysik, München.

** The National Center for Atmospheric Research is sponsored by the National Science Foundation. 


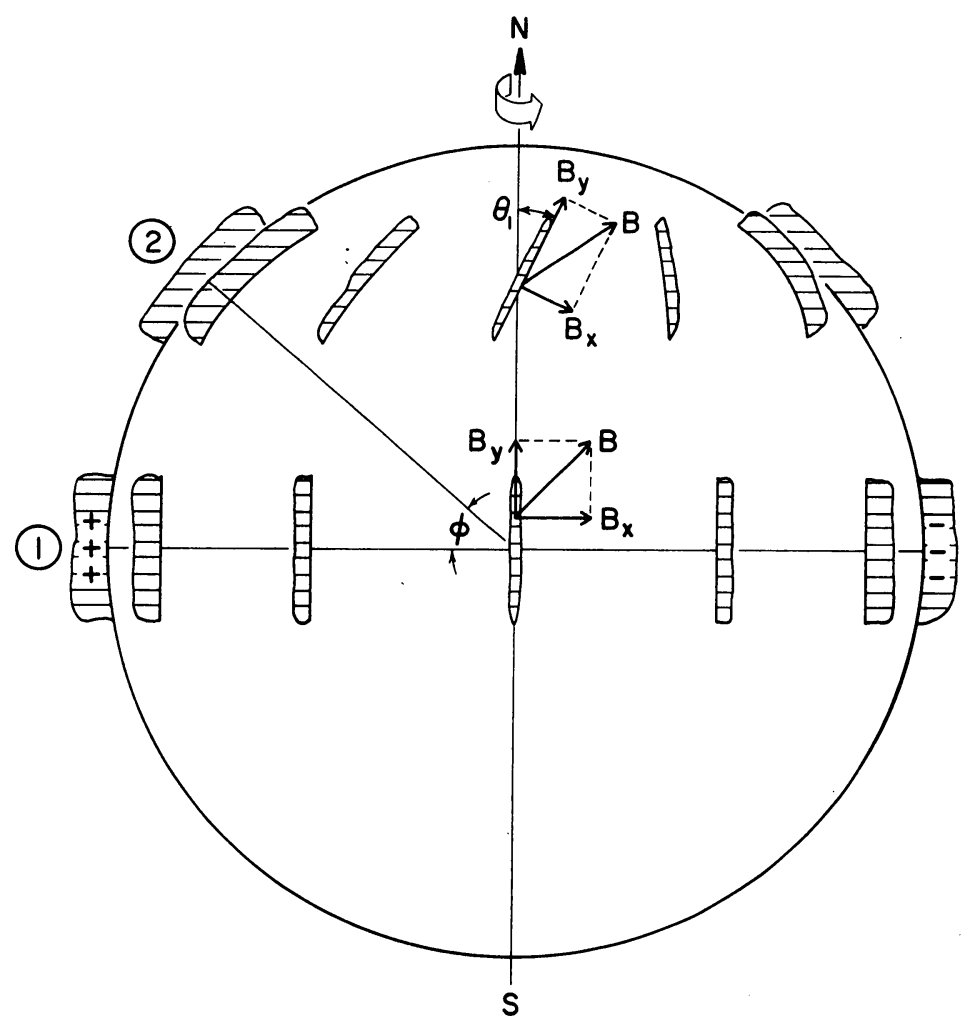

Fig. 1. Parameters defining (a) prominence in the plane of the sky on the solar equator (Prominence 1) and (b) a prominence at latitude $\varphi$ and not in the plane of the sky (Prominence 2).

which one would measure if the prominence were on the equator, is given by

$$
\tan \theta=\tan \theta_{1} \cos \phi
$$

For high-latitude prominences (the polar crown, $\phi>60^{\circ}$ ) the measured angle $\theta_{1}$ differs significantly from the angle $\theta$. Since we know $\phi$ from the observations, we could, in principle, correct our data for this effect. But for $\phi$ and $\theta_{1}$ close to $90^{\circ}$ the value of $\theta$ varies strongly with $\theta_{1}$. Since $\theta_{1}$ cannot be determined very accurately, the calculated $\theta$ would be much less accurate. Consequently, we did not correct $\theta$ according to Equation (1), but treated the observations of polar-crown filaments as a separate set of data.

\section{Relation Between the Observed Field Distribution and the True Angular Distribution}

From the observations, see Figure 2, we can calculate a mean value, $\bar{B}_{\|}$, for each $\theta$, and in principle we can describe the relationship by a function, $F_{\text {obs }}(\theta)$ say. However, of primary interest is the angle, $\alpha$, between the filament and the magnetic field, see Figure 3.

To proceed we make a number of assumptions: 


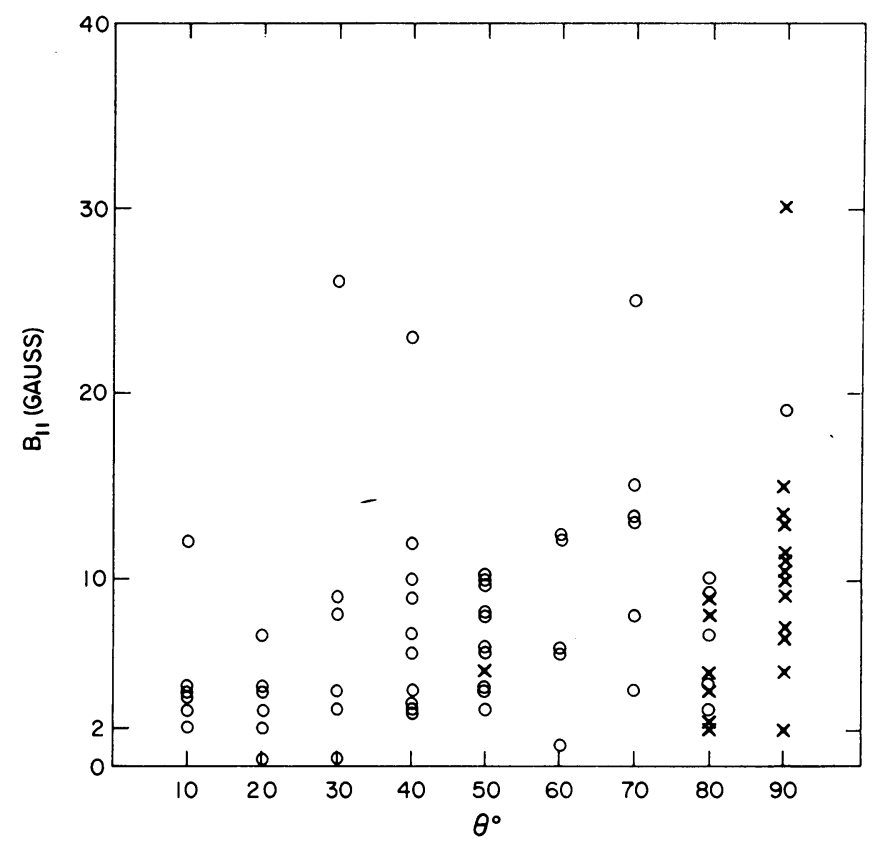

Fig. 2. The observed longitudinal component of the magnetic field, $B_{\|}$, plotted against the angle $\theta$ between the north-south direction on the Sun and the long axis of the prominence.

The crosses indicate polar crown prominences.

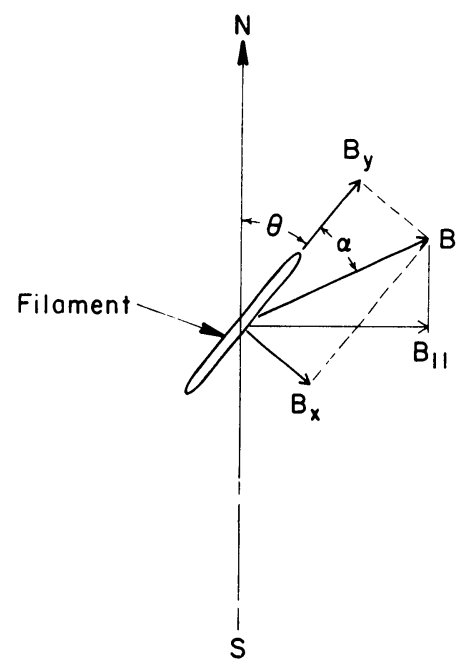

Fig. 3. The relationship between the angles $\alpha$ and $\theta$, and the components of the magnetic field vector $B$.

(i) The magnetic field is horizontal (a possible $z$ component may be added without changing the results). Then

$$
B_{\|}=B_{0}|\sin (\alpha+\theta)|
$$

where $\alpha$ and $\theta$ can take values $0 \leqslant \alpha \leqslant \pi$, and $-\pi / 2 \leqslant \theta \leqslant+\pi / 2$. 
(ii) The horizontal magnetic field vectors in quiescent prominences may be described by a distribution function, viz.

$$
\frac{N}{N_{\text {tot }}} \mathrm{d} B_{0} \mathrm{~d} \alpha=f\left(B_{0}, \alpha\right) d B_{0} \mathrm{~d} \alpha,
$$

where $N$ is the number of filaments having a field vector of strength $B_{0}$, making an angle $\alpha$ with the $y$-axis, and $N_{\text {tot }}$ is the total number of filaments. Equations (2) and (3) provide the average line-of-sight field

$$
\bar{B}_{\|}(\theta)=\int_{0}^{\infty} \int_{0}^{\pi} B_{0}|\sin (\alpha+\theta)| f\left(B_{0}, \alpha\right) \mathrm{d} \alpha \mathrm{d} B_{0} .
$$

(iii) The distribution function is separable, i.e.

$$
f\left(B_{0}, \alpha\right)=f_{1}\left(B_{0}\right) f_{2}(\alpha) .
$$

This is not correct if the field strength and the angle $\alpha$ are correlated. To the best of our knowledge, however, such a correlation has not been shown. With Equation (5) we find

$$
\bar{B}_{\|}(\theta)=\int_{0}^{\infty} B_{0} f_{1}\left(B_{0}\right) \mathrm{d} B_{0} \int_{0}^{\pi}|\sin (\alpha+\theta)| f_{2}(\alpha) \mathrm{d} \alpha=\bar{B}_{0} F(\theta) .
$$

Now, if $F(\theta)=\overline{B_{\|}(\theta) / B_{0}}$ is given from the observations, one has to determine $f_{2}(\alpha)$ such that

$$
F(\theta)=\int_{0}^{\pi}|\sin (\alpha+\theta)| f_{2}(\alpha) \mathrm{d} \alpha .
$$

(iv) The distribution is symmetric around $\alpha=\pi / 2$, or $f_{2}(\pi-\alpha)=f_{2}(\alpha)$. Then $F(\theta)$ is symmetric with respect to $\theta=0$ and

$$
F(\theta)=2\left(\cos \theta \int_{\theta}^{\pi / 2} \sin \alpha f_{2}(\alpha) \mathrm{d} \alpha+\sin \theta \int_{0}^{\theta} \cos \alpha f_{2}(\alpha) \mathrm{d} \alpha\right) .
$$

To invert this integral equation for $f_{2}(\alpha)$ we note that

$$
\frac{1}{2} F^{\prime}(\theta)=-\sin \theta \int_{\theta}^{\pi / 2} \sin \alpha f_{2}(\alpha) \mathrm{d} \alpha+\cos \theta \int_{0}^{\theta} \cos \alpha f_{2}(\alpha) \mathrm{d} \alpha,
$$

and

$$
\begin{aligned}
\frac{1}{2} F^{\prime \prime}(\theta)= & -\cos \theta \int_{\theta}^{\pi / 2} \sin \alpha f_{2}(\alpha) \mathrm{d} \alpha+\sin ^{2} \theta f_{2}(\theta)+ \\
& +\sin \theta \int_{0}^{\theta} \cos \alpha f_{2}(\alpha) \mathrm{d} \alpha+\cos ^{2} \theta f_{2}(\theta),
\end{aligned}
$$


whence

$$
f_{2}(\alpha)=\frac{1}{2}\left[F^{\prime \prime}(\alpha)+F(\alpha)\right] .
$$

Equation (9) shows that

$$
F^{\prime}(0)=0=F^{\prime}\left(\begin{array}{l}
\pi \\
2
\end{array}\right)
$$

for all functions $f_{2}$ which do not contain $\delta$-functions at $\alpha=0, \alpha=\pi / 2$. Therefore, only functions $F(\theta)$ which fulfill Equation (12) can be used in Equation (11).

\section{Numerical Examples}

It would seem a reasonable approach to try to approximate the observations by simple analytic curves $F(\theta)$ which fulfill conditions (12), and then calculate $f_{2}(\alpha)$ from Equation (11). However, for polynomials $F(\theta)$ in reasonable agreement with observations the calculated $f_{2}(\alpha)$ turns out to be not positive definite over the whole range $0 \leqslant \alpha \leqslant \pi / 2$. This means that we have to find an analytic function $F(\theta)$ which describes the observations well enough, and at the same time gives a positive definite distribution function $f_{2}(\alpha)$. Since our observations do not define $F_{\text {obs }}(\theta)$ with sufficient accuracy, this procedure amounts to trying any possible function $F(\theta)$ and calculate the corresponding $f_{2}(\alpha)$ until one finds a solution which fulfills $f_{2}(\alpha) \geqslant 0$ for $0 \leqslant \alpha \leqslant \pi / 2$.

Instead, we assume some simple distribution functions $f_{2}(\alpha)$, then calculate the $F(\theta)$ 's and compare these functions with observations. We normalize $f_{2}(\alpha)$ to give $\int_{0}^{\pi} f_{2}(\alpha) \mathrm{d} \alpha=1$, and use Equation (8) to calculate $F(\theta)$.

Case $1 \quad f_{2}(\alpha)=\frac{1}{\pi}$.

This case gives $F(\theta)=2 / \pi$.

Case $2 \quad f_{2}(\alpha)=\frac{1}{2}\left[\delta\left(\alpha-\alpha_{0}\right)+\delta\left(\alpha-\left(\pi-\alpha_{0}\right)\right)\right]$, with $0 \leqslant \alpha_{0} \leqslant \frac{\pi}{2}$.

This leads to

$$
F(\theta)=\left\{\begin{array}{lll}
\cos \theta \sin \alpha_{0} & \text { for } & 0 \leqslant \theta \leqslant \alpha_{0} \\
\sin \theta \cos \alpha_{0} & \text { for } & \alpha_{0} \leqslant \theta \leqslant \frac{\pi}{2}
\end{array}\right.
$$

Special cases are: $\alpha_{0}=0$ (the field is parallel to the filament), and $\alpha_{0}=\pi / 2$ (the field is along the $x$-axis, i.e. the Kippenhahn-Schlüter (1957) configuration).

Case $3 \quad f_{2}(\alpha)=\frac{2 n+1}{2}\left(\frac{2}{\pi}\right)^{2 n+1}\left(\frac{\pi}{2}-\alpha\right)^{2 n}$.

With increasing $n$ this gives higher and higher concentration close to $\alpha=0$. We then find

$$
F(\theta)=\frac{2 n+1}{2}\left(\begin{array}{l}
2 \\
\pi
\end{array}\right)^{2 n+1}\left[\cos \theta \int_{0}^{\pi / 2-\theta} \alpha^{2 n} \cos \alpha \mathrm{d} \alpha+\sin \theta \int_{\pi / 2-\theta}^{\pi / 2} \alpha^{2 n} \sin \alpha \mathrm{d} \alpha\right]
$$


These integrals may be solved by a recursion procedure which leads to

and $F_{0}=2 / \pi$.

$$
F_{2 n}=\frac{2 n+1}{(\pi / 2)^{2}}\left[\frac{\pi}{2}\left(1-\frac{\theta}{\pi / 2}\right)^{2 n}+2 n \sin \theta-2 n F_{2(n-1)}\right]
$$

\section{Interpretations}

From the observations, Figure 2, the average field strength for each 10 -degree interval in $\theta$ is calculated together with the root mean squares of the deviation, leaving out the four points which lie far above the bulk of the observed values. The results are plotted in Figure 4.

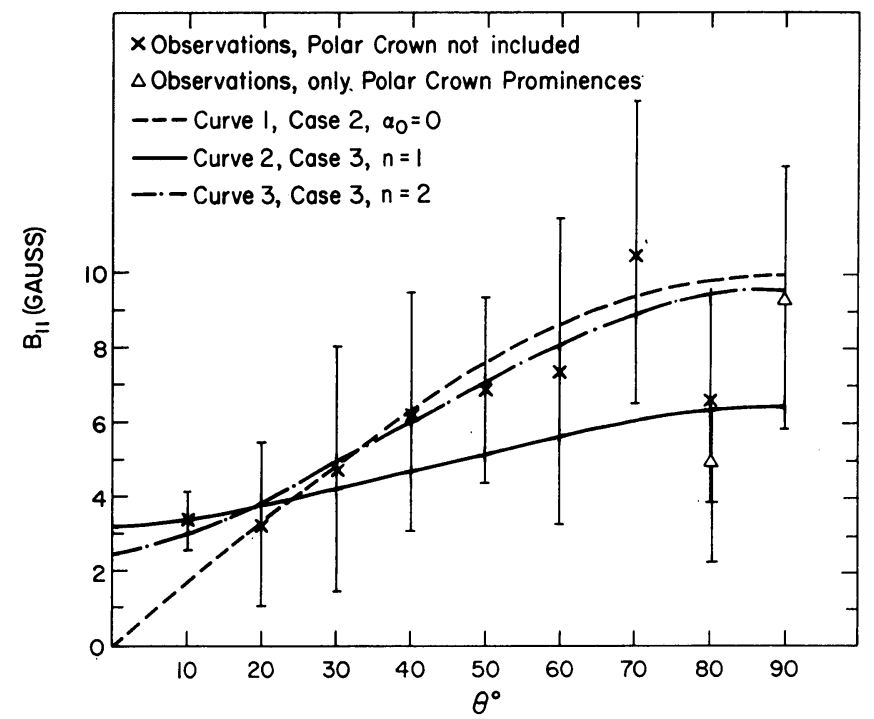

Fig. 4. Different functions, $F(\theta)$, from Equation (8) compared to the observed function $F_{\mathrm{obs}}(\theta)$.

The observations immediately rule out a distribution function which is independent of $\alpha$ (Case 1, i.e. $F(\theta)=$ const), as well as the configuration where the field is strictly perpendicular to the $y$-axis (Case 2, with $\alpha_{0}=\pi / 2$, i.e. $F(\theta) \propto \cos \theta$ ). The curves drawn in Figure 4 are (1) the field parallel to the $y$-axis (Case 2, with $\alpha_{0}=0$, i.e. $F(\theta) \propto \sin \theta$ ), (2) a quadratic distribution function (Case 3, with $n=1$ ), and (3) a fourth order distribution function (Case 3, with $n=2$ ).

Curve (1) seems to be in disagreement with the observations at small angles, $\theta \approx 10^{\circ}$. Here the observed $\bar{B}_{\|}$is about twice as large as given by curve (1). This means that the field in general is not exactly parallel to the filament. Curve (2) is much too flat, i.e. the ratio $F(\pi / 2) / F(0)$ is about a factor of two too small. The curve which fits best is curve (3), calculated from the distribution function

$$
f_{2}(\alpha)=\frac{5}{2}\left(\frac{2}{\pi}\right)^{5}\left(\frac{\pi}{2}-\alpha\right)^{4}
$$

shown in Figure 5. 


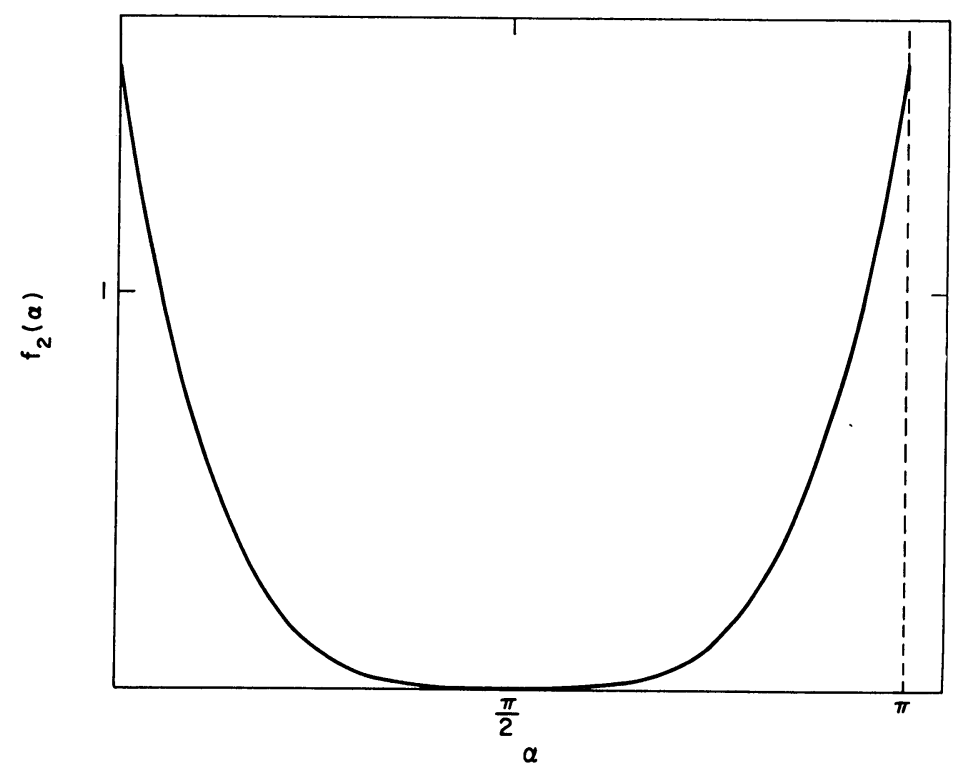

Fig. 5. The distribution function, $f_{2}(\alpha)$ from Equation (13).

Using the distribution function given by Equation (13), we can calculate the mean angle, $\bar{\alpha}$,

$$
\bar{\alpha}=\int_{0}^{\pi / 2} f_{2}(\alpha) \alpha \mathrm{d} \alpha / \int_{0}^{\pi / 2} f_{2}(\alpha) \mathrm{d} \alpha=\frac{1}{6} \frac{\pi}{2},
$$

or $\bar{\alpha} \approx 15^{\circ}$. This indicates that the magnetic field traverses the quiescent prominence sheet under a small but finite angle. From curve (3) of Figure 4 and Equation (6) we find that the average total field strength $\bar{B}_{0}$ is about $10 \mathrm{G}$.

\section{References}

Harvey, J.: 1969, Ph:D. Diss., Univ. of Colorado.

Ioshpa, B.: 1968, Results of Researches on the International Geophysical Projects, Solar Activity, No. 3, Nauka, Moscow, p. 44.

Kippenhahn, R. and Schlüter, A.: 1957, Z. Astrophys. 43, 36.

Rust, D.: 1966, Ph.D. Diss., Univ. of Colorado. 